\title{
Percutaneous balloon aortic valvuloplasty in different age groups
}

\author{
Anna Olasińska-Wiśniewska, Olga Trojnarska, Marek Grygier, Maciej Lesiak, Stefan Grajek \\ $1^{\text {st }}$ Department of Cardiology, Poznan University of Medical Sciences, Poland
}

Postep Kardiol Inter 2013; 9, 1 (31): 61-67

DOI: 10.5114/pwki.2013.34029

\begin{abstract}
Aortic stenosis is a congenital or acquired reduction in the area of the aortic valve, resulting in obstruction of the blood flow from the left ventricle to the aorta. Aortic stenosis accounts for $2-5 \%$ of all congenital heart defects and is a potentially life-threatening disorder. In adults aortic stenosis represents $34 \%$ of all valvular heart diseases. Degenerative etiology is present in $80 \%$ of cases. Patients with mild aortic stenosis are usually asymptomatic. Symptoms of the disease occur along with the disappearance of effective compensatory mechanisms. These are symptoms of low cardiac output syndrome manifested as fainting, dizziness, ischemic pains, exercise intolerance, arrhythmias with the risk of sudden cardiac death, and heart failure. As soon as the symptoms occur the prognosis significantly worsens, which is associated with a high risk of death. Percutaneous aortic valvuloplasty is a palliative method of treatment of aortic stenosis. The aim of the procedure is to relieve left ventricular outflow tract obstruction, thereby improving cardiac output. The etiology, course of the aortic stenosis and treatment methods, including invasive procedures, vary depending on the patients' age. The purpose of this paper is to present the characteristics of the aortic valve disease and the strategy of aortic balloon valvuloplasty in different age groups.
\end{abstract}

Key words: aortic stenosis, balloon valvuloplasty.

\section{Aortic valve stenosis - the nature, pathophysiology, symptoms and diagnosis of the disease}

Aortic valve stenosis is a congenital or acquired decrease of aortic valve area resulting in an impaired outflow of blood from the left ventricle into the aorta. Left ventricular afterload rises, which leads to increased end-diastolic pressure in this heart cavity [1]. Maintenance of adequate cardiac output is possible due to compensatory left ventricular hypertrophy. The balance between increased oxygen demand of hypertrophied myocardium and oxygen supply by coronary arteries becomes impaired, which is also caused by atherosclerotic changes in the coronary arteries developing with age. Hypertrophied and ischemic myocardium shows diastolic dysfunction, which is further accompanied by systolic dysfunction leading to decrease of the cardiac output and development of heart failure in more advanced cases of the disease.

Symptomatology of the disease is reflected by its pathophysiology. Patients with mild aortic valve stenosis are usually asymptomatic. Symptoms of the disease manifest along with depletion of the compensatory mechanisms and consist of symptoms of low cardiac output such as fainting and dizziness, anginal pains, exercise intolerance, life-threatening arrhythmias and heart failure in the end stage of the disease. The onset of symptoms significantly worsens the patient's prognosis as it is related to higher mortality risk (about $50-85 \%$ during 5 years from the onset of symptoms) $[2,3]$. The risk evidently increases with age as 2-year and 3 -year survival are $37-40 \%$ and $25 \%$ respectively in patients over 70 years of age $[4,5]$.

Apart from data from the medical history, the presence of typical systolic murmur with maximum loudness in the second or third right parasternal intercostal space and signs of left ventricular hypertrophy in ECG, the diagnosis of aortic valve stenosis is based mainly on echocardiographic examination. An experienced echocardiographer is able to precisely determine the anatomy of the aortic valve, which is usually fibrotic and calcified, and to assess the anatomical and hemodynamic consequences of the disease. This examination is used to guide therapeutic management including qualification for interventional or surgical treatment.

\section{Corresponding author:}

Anna Olasińska-Wiśniewska MD, PhD, 15t Department of Cardiology, Poznan University of Medical Sciences, 1/2 Długa St, 61-848 Poznan, Poland, tel.: +48 603862713 , e-mail: anna.olasinska@poczta.onet.pl

Received: 28.11.2012, accepted: 21.01.2013. 
Although the significance of the disease is similar in children and adults, its etiology, course and forms of treatment including interventional cardiology methods differ with age of the patient. For this reason the characteristics of this disease in different age groups are presented below.

\section{Congenital aortic valve stenosis}

Congenital aortic valve stenosis is responsible for $2-5 \%$ of all congenital heart diseases [6-8]. It occurs 4 times more frequenty in males $[1,8]$. Due to its significance in the development of the left ventricle it is one of the structural heart abnormalities with the worst prognosis. It may exist as an isolated pathology, but in $20 \%$ of patients the disease coexists with other diseases of the "left heart" such as coarctation of the aorta and mitral valve stenosis, together forming the complex Shone syndrome. Aortic stenosis is also a part of the Noonan and Williams syndromes [1, 7].

Advanced valvular stenosis may prevent the survival of the fetus. It may also lead to development of hypoplastic left heart syndrome (HLHS), characterized by lack of physiologic stimulation of proper development of heart structures due to decreased flow through the aortic valve, which results in hypoplasia of the mitral valve, the left ventricle and the aortic arch [8, 9]. If these dramatic fetal complications do not occur, aortic stenosis manifests as rapidly progressing left ventricular failure directly after birth. The clinical stage depends on the presence of intracardiac shunts, including mainly persistent arterial duct. This conduit transfers most of the blood to the systemic circulation and its closure leads to rapid worsening of the clinical status of the neonate [8].

In infants in whom the degree of impairment of the aortic valve structure and function prevent survival, it is necessary to immediately analyze the clinical condition and to determine further therapeutic actions.

In normal conditions the aortic valve consists of three leaflets moving away symmetrically from each other during left ventricular systole, which causes blood flow out of the ventricle and around the "swallow's nests". Any change of the valvular anatomy including the presence of two symmetrical cusps, three improperly constructed cusps, often forming a "half and two quarters" structure with frequent atresia of the connecting commissures, or the presence of only one cusp in extreme cases, results in the situation where every heart contraction has direct and longterm complications. The strike of the blood stream against the ventricular surface of the valve leads to its opening and therefore every heart contraction causes an injury of the valve inevitably resulting in its degeneration. Another factor increasing the pressure overload of the child's left ventricle is frequent coexistence of annular hypoplasia or less frequently aortic arch hypoplasia. Worsening of symptoms and prognosis depend on the severity of the described changes and on the available methods of treatment [7].

\section{Aortic stenosis in adults}

According to the European registry of heart diseases [10] including 5001 patients from 25 countries, aortic valve stenosis is responsible for $34 \%$ of all valvular heart diseases. In $80 \%$ of patients it is caused by degenerative changes often involving a bicuspid valve, which should be taken into consideration when choosing an invasive treatment [1, 2]. The progress of civilization in the so-called developed countries over the last several decades has significantly reduced the incidence of rheumatic heart disease. This is why it is currently responsible for only $11 \%$ of all cases of aortic stenosis $[1,2]$.

The surgical valve replacement is an established and recommended method of treatment of patients with symptomatic aortic valve stenosis [2]. It has been performed since 1961 , leading to significant decrease of mortality $(74.9 \%$ of patients survive 15 years) [11]. One-year, two-year and fiveyear survival in octogenarians is $87 \%, 78 \%$ and $68 \%$ respectively [5]. Quality of life of the patients is also improved $[2,11]$. Obviously, the results of such treatment depend on the stage of the disease, concomitant diseases and the age of the patient undergoing surgery. The risk of periprocedural death in patients below 70 years is $1-3 \%$, while in octogenarians with significant concomitant diseases it increases to $5-15 \%[2,12]$. This is the reason why approximately $1 / 3$ of patients above 80 years of age are disqualified from the procedure $[2,13]$.

\section{Aortic valve stenosis during pregnancy}

Aortic valve stenosis during pregnancy is a serious therapeutic problem. The severity of the disease is increased by hemodynamic changes resulting from this state such as an increase of cardiac output with decrease of systemic resistance. Therefore a young, often asymptomatic patient may present typical, dangerous manifestations of the disease described above [14]. Severe aortic valve stenosis is considered as the highest risk condition of serious cardiological complications and death during pregnancy and childbirth [14].

\section{Percutaneous balloon aortic valvuloplasty \\ - the nature of the procedure, its technique, indications in different age groups and complications}

Percutaneous balloon aortic valvuloplasty is performed to dilate the narrowed valve. It is a palliative procedure both in children and in adults as it decreases the valvular stenosis, but permanent elimination of the transaortic gradient is impossible. This procedure however may postpone the definitive surgery or at least improve the patient's critical condition.

The technical aspects of the procedure are similar in all age groups. Aortic valvuloplasty consists of the introduction of the balloon catheter into the stenosed aortic valve followed by mechanical dilation of the valve caused by bal- 
loon inflation [6]. The procedure is often performed using the Seldinger technique with a retrograde approach through the femoral or carotid artery or umbilical artery in a fetus $[1,6,15]$. Some centers prefer the approach through the femoral vein in children and in exceptional situations through the umbilical vein and interatrial septum in neonates [15]. Aortography and blood pressure assessment in the aorta are followed by the introduction of a diagnostic catheter into the left ventricle, which is used to register pressure in the ventricle. Subsequently a guidewire with a soft tip is placed in the left ventricle, which is used to introduce a balloon catheter for valvuloplasty. Balloon inflation in the stenosed valve leads to its dilation. The balloon has a $3.5 \mathrm{~mm}$ diameter in children and $35 \mathrm{~mm}$ in adults and its dimension should not exceed more than 1.1-fold the size of the aortic annulus [1]. It is important to obtain a stable balloon position during its inflation. This is currently achieved by rapid stimulation at $160-220 \mathrm{bpm}$ or administration of adenosine to temporarily stop outflow of blood from the left ventricle [3]. Control pressure assessment in the ventricle and in the aorta as well as aortography after the procedure help to determine the effect of the procedure and the degree of regurgitation. For an optimum effect, it is often necessary to inflate the balloon several times.

As demonstrated by the characteristics of patients with aortic valve stenosis, specific indications for balloon aortic valvuloplasty differ in children and in adults.

\section{Balloon aortic valvuloplasty in fetuses}

Balloon valvuloplasty in fetuses is not intended for treatment of the disease, but to prevent worsening heart failure and to increase blood flow through the heart to counteract the hemodynamic changes leading to the onset of hypoplastic left heart syndrome [16-19]. The first two attempts at such procedures were performed in London by the team of Maxwell in 1991 [16]. Unfortunately the treatment did not save the children - in the first child it was impossible to cross the aortic valve with a catheter and in the second one the intrauterine procedure failed was performed twice. The procedure was reattempted after birth, but the child died due to left ventricular dysfunction caused by endocardial fibroelastosis [16]. Despite these disappointing results, the method was developed further and over 100 prenatal procedures have been performed so far. The most experienced centers are located in Boston (the team of McElhinney and Tworetzy) [17] and in Linz in Austria directed by Professor Arzt [18]. Several initial procedures have also been conducted in Warsaw by Professor Dangel [19]. There is no doubt that the execution of balloon valvuloplasty in the prenatal period is technically extremely difficult and related to a high probability of failure as well as a significant risk of serious complications. It is also not always possible to improve the size and function of the left ventricle [17]. Extremely important features include proper patient selection and timing of the procedure. For these reasons indications for balloon valvu- loplasty in fetuses are not yet well established. We only know that the procedure may be performed in fetuses in which lack of intervention will likely lead to death or to the development of the hypoplastic left heart syndrome [19].

\section{Balloon aortic valvuloplasty in neonates and in children}

In 1983 Lababidi et al. [20, 21] for the first time performed and described the balloon valvuloplasty procedure in 23 children from 2 to 17 years of age. A positive effect of the procedure manifesting as decrease of the maximal transaortic gradient (from $113 \pm 48 \mathrm{~mm} \mathrm{Hg}$ to $32 \pm 15 \mathrm{~mm} \mathrm{Hg}$ ) was obtained in all patients. Directly after the procedure mild aortic regurgitation was found in 10 patients and the remaining 13 patients did not present any reverse flow. The operator's experience and technological progress enable these procedures to be performed in younger patients, including neonates $[22,23]$.

In this youngest group of patients with critical aortic valve stenosis, balloon valvuloplasty is currently a treatment of choice. Persistent patency of arterial duct arteriosus is a prerequisite for the maintenance of adequate cerebral and coronary perfusion until the time of the procedure. If it is impossible to perform the biventricular procedure, a Norwood procedure is done (a description of this is beyond the scope of this paper).

Surgical commissurotomy is an alternative method of aortic valve enlargement in children beyond the neonatal period and early infancy. It will be described later in this paper. Performance of any of these procedures in advanced aortic stenosis enables normal development of the left ventricle and avoidance of complications typical for this disease until the time it is possible to perform a definitive correction. Some young patients undergo the Ross procedure, consisting of implantation of a native pulmonary valve in the aortic position and replacement of the pulmonary valve with a homograft. This procedure has many advocates, as it enables artificial materials to be avoided, but its opponents argue that a disease of one valve is replaced with a disease of two valves in the later phase. Therefore, in most cases, after the patient has reached the weight and dimensions of an adult, implantation of a biological or mechanical prosthesis is performed. Balloon valvuloplasty of the aortic valve has therefore a key therapeutic significance in the youngest patients.

Currently, the early results of balloon valvuloplasty in children are good, because the procedure can be performed in $80-90 \%$ of them with a periprocedural mortality of $4-9 \%$ [22-25]. The result of the procedure is influenced by adequate patient selection, left ventricular function assessment and occurrence of endocardial fibroelastosis [26]. Decrease of the afterload after a successful procedure in children with the low cardiac output syndrome does not cause an effective left ventricular systole and in consequence does not lead to a satisfactory effect of valvuloplasty [16]. However, performance of this procedure may have beneficial effects 
in the case of aortic valve annulus hypoplasia and left ventricular hypoplasia by creation of conditions for normalization of the dimensions of "left heart" structures at 1 year after the procedure $[25,27]$.

The long-term results of balloon valvuloplasty in children are good. Currently, survival reaches $95 \%, 89-93 \%$ and $81-88 \%$ after 5,10 and 20 years, respectively [28, 29]. In a multicenter analysis of 1004 patients Ewert et al. [22] demonstrated that $50 \%$ of children with congenital aortic valve stenosis treated with this method do not require surgical aortic valve replacement within 10 years after the procedure and that $90 \%$ of them remain in good clinical condition with normal left ventricular function during that period. The mid-term and the long-term results of treatment are worse in neonates and infants than in children above the first year of life, because, on the former, such procedures are often performed for emergency indications caused by more advanced disease. Balmer et al. [23] observed that reintervention was required in $52 \%$ of children who underwent the procedure before the $3^{\text {rd }}$ month of life and in $25 \%$ of children who underwent the procedure after this period. The same authors [23] report that younger patients had a higher risk of femoral artery closure (57\% vs. $18 \%)$, most likely due to technical reasons. The necessity of more aggressive dilation of a significantly restrictive valve (advanced dysmorphia, frequent aortic annulus hypoplasia) in neonates and younger infants was most likely the cause of more frequent, progressing in time, aortic regurgitation in comparison to older infants. After 1 year this complication was present in $40 \%$ of patients and after 2 years in $50 \%$ of patients operated on before the third month of life. However, if the procedure was performed at an older age, aortic regurgitation was found in $30 \%$ of patients after 1 year and in $40 \%$ after 2 years. The authors reported that the periprocedural death of 3 patients and the death of 3 patients in the long term was higher in the group of younger children in comparison to older children, where only one long-term death was observed. This difference was probably related to the patient's pre-procedural condition [23]. The studies of Ewert et al. [22] conducted on patients between the $1^{\text {st }}$ day of life and 18 years of age confirm the fact that the frequency of complications including arrhythmia and injury of the valve or the arteries decreases with age. They were present in 15\% of neonates, $11 \%$ of infants and $6 \%$ of older children. The analysis of the results of balloon aortic valvuloplasty showed that the most frequent complication is valvular regurgitation, which occurs in the acute form in $15 \%$ of treated patients $[6,27]$. It is caused by excessive deformation of the cusps and rupture of the commissures $[6,27]$. Progressive degenerative changes lead to increase of reverse flow volume. In consequence, chronic aortic regurgitation is found in $22-35 \%$ of patients $5-14$ years after the procedure $[6,27-30]$. The use of too small balloon may not lead to expected results and may leave residual valvular stenosis. The cause of this phenomenon is also related to the anatomy of the disease including, of course, aortic annulus hypoplasia. It was observed that this complication occurs more frequently if the procedure is performed before the $3^{\text {rd }}$ year of life. It was also demonstrated that the symptoms caused by restenosis occur more frequently and are more severe if the postprocedural maximal transaortic gradient exceeded $30 \mathrm{~mm} \mathrm{Hg}$ [27, 30, 31]. According to Demkow et al. [31], a postprocedural transaortic gradient exceeding $40 \mathrm{~mm} \mathrm{Hg}$ increases the risk of significant restenosis and reintervention six-fold. Interpretation of the potential increase of transaortic gradient at long term should include the beneficial phenomenon of left ventricular systolic function improvement caused by the procedure. Repeated percutaneous valvuloplasty or surgery is necessary in $30-44 \%$ of patients [22, 27, 28, 31, 32]. As mentioned, the last stage of aortic stenosis treatment consists of radical surgical aortic valve replacement.

Before the era of percutaneous procedures aortic valvuloplasty was performed with the use of surgical methods. This procedure is performed via sternotomy. After heparin administration, cannulation of the aorta and the right atrium and initiation of extracorporeal circulation at normothermia or at moderate hypothermia, the aortic bulb is opened and incisions of the valve commissures are made under visual control [8]. The advantage of surgical valvulotomy is a possibility to operate under visual control and the disadvantages include the procedure's difficulty and the risk related to reoperation in case of reintervention required in the future [9]. A comparison of the efficacy of these two methods of treatment demonstrates that balloon valvuloplasty is related to higher, approximating $18 \%$, incidence of significant aortic regurgitation, while the incidence of this complication after surgery is only $3 \%$. Cardiac surgery is related to more frequent (mean 1.8 times) residual stenosis [26]. However, such comparisons are difficult, because the procedures were performed at different times (interventional treatment was introduced 30 years later than surgical treatment) and there were different possibilities of periprocedural and postprocedural management. It is always important to select patients for the procedure properly. Today, percutaneous intervention is most often used in urgent situations. Patients with advanced stenosis caused by unicuspid or bicuspid valve and fused commissures should be referred for cardiac surgery because of the significantly higher risk of their injury during balloon valvuloplasty [32].

\section{Balloon aortic valvuloplasty in adults}

The first balloon aortic valvuloplasty procedures in adults were performed by the team of Cribier et al. in 1986 [33]. The results of the procedures conducted on 92 patients between 38 and 91 years of age were very promising; maximal transaortic gradient dropped from $75 \pm 26 \mathrm{~mm} \mathrm{Hg}$ to $30 \pm 13 \mathrm{~mm} \mathrm{Hg}$ and the aortic orifice area increased from $0.49 \pm 0.17 \mathrm{~cm}^{2}$ to $0.93 \pm 0.36 \mathrm{~cm}^{2}$ [34]. In-hospital mortality was $3.3 \%$ and 8 other patients died during the follow- 
ing 3 months ( $9.8 \%$ of patients). Other patients presented significant, subjective clinical improvement and most of the patients (90\%) were in NYHA class I or II. Based on the described results of treatment the authors suggested that the proposed method of aortic valve dilation with a balloon is a simple alternative to valve replacement in elderly patients at high periprocedural risk [33, 34]. Economic aspects of the procedure such as low costs of the equipment, short hospitalization of several days and the lack of necessary long-term rehabilitation after the procedure were also emphasized [34]. There are even reports in the literature on the use of transluminal aortic valvuloplasty in cases of extremely advanced aortic stenosis. In 1994 Moreno et al. [35] described the performance of balloon valvuloplasty in 21 patients in cardiogenic shock. Twelve of the treated patients (57\%) survived the procedure. They had a significant, but short-term clinical improvement. Doquet et al. [36] reported the results of the aortic valve replacement preceded by valvuloplasty in 25 patients initially disqualified from the surgery because of their poor clinical condition. Four patients in this group were in cardiogenic shock and 17 presented a severe decompensated heart failure. Balloon valvuloplasty permitted sufficient stabilization of clinical condition before the final surgery, which was performed in all patients within 8-14 weeks. One patient died after the surgery and 9 patients did not survive the 5 -year observation period. Continuation of these reconstructive procedures demonstrated that the effects of balloon valvuloplasty in this group of patients are not as satisfactory as initially thought. It was shown that it is only possible to decrease the degree of stenosis, but the hemodynamically significant disease persists [37]. The results of a large registry including 674 patients undergoing balloon valvuloplasty [38] revealed that transvalvular gradient drops by half directly after the procedure and that effective aortic orifice area increases by approximately $65 \%$, but usually does not exceed $1 \mathrm{~cm}^{2}$. The stenosis increases again in the following months. Otto et al. [37] observed recurrence of stenosis as soon as 6 months after the procedure (decrease of mean effective orifice area from $0.78 \pm 0.31 \mathrm{~cm}^{2}$ measured after the procedure to $0.65 \pm 0.25 \mathrm{~cm}^{2}$ after 6 months), which led to worsening of clinical condition and the need for hospitalization within 6-12 months after the procedure. Restenosis may also occur within a few days or weeks after the procedure, which is why some of the operators suggest a repeated procedure [39-41]. Agarwal et al. [39] reported 1 -year survival of $58 \%$ of patients undergoing a single procedure and $84 \%$ of those undergoing multiple valvuloplasty procedures. It is clear, however, that further treatments multiply the risk of complications; hence their repetition is not recommended by all [42]. Complications of balloon dilation of the aortic valve are in fact not uncommon. In 20-25\% of cases they occur in the periprocedural period, usually within the first $24 \mathrm{~h}$ after the procedure [38, 43, 44]. Cardiovascular complications are the most common and usu- ally include arterial wall injury, pseudoaneurysm, arteriovenous fistula, hematoma (4.6-7\% of patients) [38, 45, 46], hypotension (8\%) [38], arrhythmias requiring treatment (10\%) [38], atrioventricular block requiring pacemaker implantation $(1-4 \%)[38,44]$ and renal function impairment manifested by increase of serum creatinine level of more than $50 \%$ (11\%) [45]. Presenting a significant risk to the life of the patient, but less frequent complications, are stroke (1-2\%), myocardial infarction (1\%), cardiac tamponade (1\%) and significant acute aortic regurgitation (1\%) [38, 39, $43,45]$. The operator's experience and technological progress have significantly improved the safety of the procedure $[36,45,47,48]$, as exemplified by the decline in the incidence of serious vascular complications from $13.5 \%$ in the 1990 s to $4.6-7 \%$ observed currently [38, 39, 45, 46]. As a result, the periprocedural mortality is only approx. 1-5.6\% $[11,38,45,48]$. The rate is higher in the long-term followup. There is an estimated survival of $50-68 \%$ after 6 months, $33-64 \%$ after 1 year and $18-37 \%$ after 3 years [37, 44, 45, 48-50]. For comparison, $50 \%$ of patients with symptomatic severe aortic stenosis treated only pharmacologically die within 2 years of observation [4, 5]. Obviously the prognosis worsens with age, but the 1-, 2- and 5-year survival of octogenarians treated conservatively reaches $52 \%, 40 \%$ and $22 \%$ respectively [5] and is not significantly different from the prognosis observed in the group of patients treated with balloon angioplasty, which proves that this treatment is merely palliative. This strategy is an attractive form of treatment enabling future therapeutic actions. However, its use is only possible in carefully selected patients. Contraindications to balloon valvuloplasty include moderate and severe aortic valve regurgitation, the presence of thrombus in the left ventricle, endocarditis and the inability to use anti-thrombotic therapy in the periprocedural period [24]. The procedure is also limited by the size of the valve annulus, which may be disproportionate to the size of the available balloons or peripheral arterial disease preventing vascular access.

In recent years there has been a renewed interest in percutaneous balloon valvuloplasty due to the rapid progress of percutaneous techniques of aortic valve prosthesis implantation. This procedure is an integral part of TAVI (transcatheter aortic valve implantation) enabling preparation of the native valve for prosthesis implantation. A separate indication is balloon valvuloplasty in pregnant women with severe aortic valve stenosis. Surgical valve replacement or balloon valvuloplasty (which is safer) should be considered if a stable clinical condition is not achieved despite intensive pharmacological therapy, the aortic orifice area is close to $1.0 \mathrm{~cm}^{2}$ and there is a maximal transaortic gradient $>75 \mathrm{~mm} \mathrm{Hg}$ and/or significant left ventricular function impairment (ejection fraction < 55\%). Because of the potential adverse effect of contrast on the fetal thyroid, it is recommended, if possible, not to perform this kind of procedure before the third trimester of pregnancy [14]. The idea of balloon valvuloplasty as an alternative to surgical aor- 
tic valve replacement did not stand the test of time, but its use is still justified. According to current guidelines [2] the use of this procedure should be limited to selected patients who can thus achieve an improvement of hemodynamic status and possibly qualify for further invasive treatment (class $\mathrm{Ilb}$, level of evidence C). Therefore, percutaneous aortic valvuloplasty is performed only as a bridging therapy in patients who are in the advanced stage of the disease and are hemodynamically unstable. It may enable one to obtain sufficient enough hemodynamic and clinical stability to consider patient qualification for aortic valve replacement or TAVI. Balloon valvuloplasty may also be considered in patients who require urgent noncardiac surgery or in individual cases as a palliative treatment when due to severe concomitant diseases cardiac surgery is contraindicated [2].

Percutaneous valvuloplasty is a method of palliative treatment of aortic valve stenosis. It can be used in all age groups of patients, from the fetus to old age. The primary indication for its use is to achieve hemodynamic and clinical improvement enabling further radical therapy of the disease.

\section{References}

1. Zipes DP, Libby P, Bonow RO, Braunwald E. Braunwald's heart disease: a textbook of cardiovascular medicine. 7th ed. Elsevier Saunders, Philadelphia 2005.

2. Authors/Task Force Members, Vahanian A, Alfieri O, Andreotti F, et al. Guidelines on the management of valvular heart disease (version 2012): The Joint Task Force on the Management of Valvular Heart Disease of the European Society of Cardiology (ESC) and the European Association for Cardio-Thoracic Surgery (EACTS). Eur Heart J 2012; 33: 2451-2496.

3. Leon MB, Smith CR, Mack M, et al.; PARTNER Trial Investigators. Transcatheter aortic-valve implantation for aortic stenosis in patients who cannot undergo surgery. N Engl J Med 2010; 363: 1597-1607.

4. O'Keefe JH Jr, Vlietstra RE, Bailey KR, Holmes DR Jr. Natural history of candidates for balloon aortic valvuloplasty. Mayo Clin Proc 1987; 62: 986-991.

5. Varadarajan P, Kapoor N, Bansal RC, Pai RG. Survival in elderly patients with severe aortic stenosis is dramatically improved by aortic valve replacement: results from a cohort of 277 patients aged $>$ or $=80$ years. Eur J Cardiothorac Surg 2006; 30: 722-277.

6. Brzezińska-Rajszys G, Dąbrowski M, Rużyłło W, Witkowski A. Interventional cardiology [Polish]. Wydawnictwo Lekarskie PZWL, Warsaw 2009.

7. Respondek-Liberska M. Prenatal cardiology for obstetricians and pediatric cardiologists [Polish]. Czelej, Lublin 2006.

8. Skalski J, Religa Z. Kardiochirurgia dziecięca. Wydawnictwo Śląsk, Katowice 2003.

9. Alsoufi B, Karamlou T, McCrindle BW, Caldarone CA. Management options in neonates and infants with critical left ventricular outflow tract obstruction. Eur J Cardiothorac Surg 2007; 31: 1013-1021.

10. lung B, Baron G, Butchart EG, et al. A prospective survey of patients with valvular heart disease in Europe: The Euro Heart Survey on Valvular Heart Disease. Eur Heart I 2003; 24: 1231-1243.

11. Kvidal P, Bergström R, Hörte LG, Ståhle E. Observed and relative survival after aortic valve replacement. J Am Coll Cardiol 2000; 35: 747-756.

12. Vahanian A, Otto CM. Risk stratification of patients with aortic stenosis. Eur Heart J 2010; 31: 416-23.
13. lung B, Cachier A, Baron G, et al. Decision-making in elderly patients with severe aortic stenosis: why are so many denied surgery? Eur Heart J 2005; 26: 2714-2720.

14. Authors/Task Force Members, Regitz-Zagrosek V, Blomstrom Lundqvist C, Borghi C i wsp. ESC Guidelines on the management of cardiovascular diseases during pregnancy: the Task Force on the Management of Cardiovascular Diseases during Pregnancy of the European Society of Cardiology (ESC). Eur Heart J 2011; 32: 3147-3197.

15. Kusa J. Balloon valvuloplasty of critical aortic stenosis performed from an umbilical access in a neonate with endocardial fibroelastosis: a case report. Kardiol Pol 2006; 64: 415-418.

16. Maxwell D, Allan L, Tynan MJ. Balloon dilatation of the aortic valve in the fetus: a report of two cases. Br Heart J 1991; 65: 256-258.

17. McElhinney DB, Marshall AC, Wilkins-Haug LE, et al. Predictors of technical success and postnatal biventricular outcome after in utero aortic valvuloplasty for aortic stenosis with evolving hypoplastic left heart syndrome. Circulation 2009; 120: 1482-1490.

18. Arzt W, Wertaschnigg D, Veit I, et al. Intrauterine aortic valvuloplasty in fetuses with critical aortic stenosis: experience and results of 24 procedures. Ultrasound Obstet Gynecol 2011; 37: 689-695.

19. Dangel J, Debska M, Koleśnik A, et al. The first successful fetal aortic balloon valvuloplasty in Poland. Ginekol Pol 2011; 82: 632-636.

20. Lababidi Z. Aortic balloon valvuloplasty. Am Heart J 1983; 106: 751-752.

21. Lababidi Z, Wu JR, Walls JT. Percutaneous balloon aortic valvuloplasty: results in 23 patients. Am J Cardiol 1984; 53: 194-197.

22. Ewert P, Bertram H, Breuer J, et al. Balloon valvuloplasty in the treatment of congenital aortic valve stenosis: a retrospective multicenter survey of more than 1000 patients. Int J Cardiol 2011; 149: 182-185.

23. Balmer C, Beghetti M, Fasnacht $M$, et al. Balloon aortic valvoplasty in paediatric patients: progressive aortic regurgitation is common. Heart 2004; 90: 77-81.

24. Braunwald E. Harrison's advances in cardiology [Polish]. Czelej, Lublin 2005.

25. Petit CJ, Ing FF, Mattamal R, et al. Diminished left ventricular function is associated with poor mid-term outcomes in neonates after balloon aortic valvuloplasty. Catheter Cardiovasc Interv 2012; 80: 1190-1199.

26. McCrindle BW, Blackstone EH, Williams WG, et al. Are outcomes of surgical versus transcatheter balloon valvotomy equivalent in neonatal critical aortic stenosis? Circulation 2001; 104: |152-|158.

27. McElhinney DB, Lock JE, Keane JF, et al. Left heart growth, function, and reintervention after balloon aortic valvuloplasty for neonatal aortic stenosis. Circulation 2005; 111: 451-458.

28. Brown DW, Dipilato AE, Chong EC, et al. Aortic valve reinterventions after balloon aortic valvuloplasty for congenital aortic stenosis intermediate and late follow-up. J Am Coll Cardiol 2010; 56: 1740-1749.

29. Maskatia SA, Ing FF, Justino $\mathrm{H}$, et al. Twenty-five year experience with balloon aortic valvuloplasty for congenital aortic stenosis. Am J Cardiol 2011; 108: 1024-1028.

30. Galal O, Rao PS, Al-Fadley F, Wilson AD. Follow-up results of balIoon aortic valvuloplasty in children with special reference to causes of late aortic insufficiency. Am Heart J 1997; 133: 418-427.

31. Demkow M, Ruzyllo W, Ksiezycka E, et al. Long-term follow-up results of balloon valvuloplasty for congenital aortic stenosis: predictors of late outcome. J Inv Cardiol 1999; 11: 220-226.

32. Reich O, Tax P, Marek J, et al. Long term results of percutaneous balloon valvoplasty of congenital aortic stenosis: independent predictors of outcome. Heart 2004; 90: 70-76. 
33. Cribier A, Savin T, Saoudi N, et al. Percutaneous transluminal valvuloplasty of acquired aortic stenosis in elderly patients: an alternative to valve replacement? Lancet 1986; 1: 63-67.

34. Cribier A, Savin T, Berland J, et al. Percutaneous transluminal balloon valvuloplasty of adult aortic stenosis: report of 92 cases. J Am Coll Cardiol 1987; 9: 381-386.

35. Moreno PR, Jang IK, Newell JB, et al. The role of percutaneous aortic balloon valvuloplasty in patients with cardiogenic shock and critical aortic stenosis. J Am Coll Cardiol 1994; 23: 1071-1075.

36. Doquet F, Godin M, Lebreton G, et al. Aortic valve replacement after percutaneous valvuloplasty: an approach in otherwise inoperable patients. Eur J Cardiothorac Surg 2010; 38: 394-399.

37. Otto CM, Mickel MC, Kennedy JW, et al. Three-year outcome after balloon aortic valvuloplasty. Insights into prognosis of valvular aortic stenosis. Circulation 1994; 89: 642-650.

38. Percutaneous balloon aortic valvuloplasty. Acute and 30-day follow-up results in 674 patients from NHLBI Balloon Valvuloplasty Registry. Circulation 1991; 84: 2383-2397.

39. Agarwal A, Kini AS, Attanti S, et al. Results of repeat balloon valvuloplasty for treatment of aortic stenosis in patients aged 59 to 104 years. Am J Cardiol 2005; 95: 43-47.

40. Waller BF, McKay C, VanTassel JW, et al. Catheter balloon valvuloplasty of stenotic aortic valves. Part I: Anatomic basis and mechanisms of balloon dilation. Clin Cardiol 1991; 14: 836-846.

41. Koning R, Cribier A, Asselin C, et al. Repeat balloon aortic valvuloplasty. Cathet Cardiovasc Diagn 1992; 26: 249-254.

42. Feldman T, Glagov S, Caroll J. Restenosis following successful balloon valvuloplasty: bone formation in aortic valve leaflets. Catheter Cardiovasc Interv 1993; 29: 1-7.
43. Vahanian A, Palacios IF. Percutaneous approaches to valvular disease. Circulation 2004; 109: 1572-1579.

44. O'Neill WW. Predictors of long-term survival after percutaneous aortic valvuloplasty: report of the Mansfield Scientific Balloon Aortic Valvuloplasty Registry. J Am Coll Cardiol 1991; 17: 193-198.

45. Ben-Dor I, Pichard AD, Satler LF, et al. Complications and outcome of balloon aortic valvuloplasty in high-risk or inoperable patients. JACC Cardiovasc Interv 2010; 3: 1150-1156.

46. Dworakowski R, Bhan A, Brickham B, et al. Effectiveness of balloon aortic valvuloplasty is greater in patients with impaired left ventricular function. Int J Cardiol 2011; 150: 103-105.

47. Anwaruddin S, Herrmann HC. Old dog, new tricks: redefining the role for balloon aortic valvuloplasty in the transcatheter aortic valve replacement era. J Invasive Cardiol 2012; 24: 63.

48. Klein A, Lee K, Gera A, et al. Long-term mortality, cause of death, and temporal trends in complications after percutaneous aortic balloon valvuloplasty for calcific aortic stenosis. J Interv Cardiol 2006; 19: 269-275.

49. Lieberman EB, Bashore TM, Hermiller JB, et al. Balloon aortic valvuloplasty in adults: failure of procedure to improve long-term survival. J Am Coll Cardiol 1995; 26: 1522-1528.

50. Tissot CM, Attias D, Himbert D, et al. Reappraisal of percutaneous aortic balloon valvuloplasty as a preliminary treatment strategy in the transcatheter aortic valve implantation era. Eurointervention 2011; 7: 49-56. 